The question of the utility of oral tradition was discussed at length. In this connexion the Conference was impressed by the rigorous rules drawn up by Dr. J. Vansina for the collection and interpretation of oral evidence, and came to the unanimous conclusion that work of this kind should be encouraged in every way possible.

Most of the papers contributed to the Conference illustrated the new concern with a more balanced view of African history by emphasizing the importance of the reaction of African societies to the impact of European formal and informal empire; by making use of hitherto unexploited documentary sources, Islamic sources in particular; by making use of oral tradition; by re-examining sources already used with different questions in mind, and so on. It is hoped that the papers-together with an editorial abstract of the main points raised in discussion-will be published in some form.

[Communicated by Mr. Terence Ranger]

\title{
Staff Problems in Tropical Countries
}

THE International Institute of Differing Civilizations held its 32 nd study session in Munich from 19 to 22 September, on the subject of 'Staff Problems in Tropical and Sub-Tropical Countries'. The discussions were introduced by five general rapporteurs: M. Georges Balandier (France), Director of Studies at the École Pratique des Hautes Études (social aspect); Dr. J. S. Coleman (U.S.A.), Professor of Political Science in the University of California (legal aspect); Sir Sydney Caine (U.K.), Director of the London School of Economics (economic aspect); M. Gabriel d'Arboussier (Mali), former President of the Grand Conseil de l'Afrique Occidentale Française (political aspect), and M. Charles Ammoun (Lebanon), Lebanese delegate at UNESCO (cultural aspect). These reports were based on some thirty regional papers from the main Asian, African, and American countries. Discussions were led by Dr. Wolfgang Pohle, President of INCIDI.

\section{L'Institut des Sciences Economiques et Commerciales Appliquées de l'Afrique Noire (Dakar)}

L'Instritut des Sciences Économiques et Commerciales Appliquées de l'Afrique Noire a été créé en 1959. C'est un Institut d'Université dirigé par un professeur de la Faculté de Droit et des Sciences Économiques de Dakar, qu'assiste un Conseil de Perfectionnement placé sous la présidence du Recteur de l'Université. Les buts de l'Institut sont l'enseignement, la recherche, et la liaison avec les Institutions étrangères et les organisations internationales. $\mathrm{La}$ tâche $\mathrm{la}$ plus urgente était relative à l'enseignement. En effet, le personnel économique et commercial d'origine africaine est encore peu nombreux, et l'Indépendance en appelle l'accroissement accélété. L'Université ne peut fournir, et pour longtemps encore, qu'une quantité restreinte des spécialistes en question.

Il y a deux sections de l'Université. La première, dite Section Économique et Commerciale, s'efforce d'apporter à nos auditeurs un complément de formation leur permettant d'accéder à des fonctions supérieures. La seconde, dite Section Statistique, d'un niveau plus relevé, vise à former des statisticiens qualifiés. Deux nouvelles séries seraient ajoutées l'année prochaine. La première, s'adressant aux diplômés de la Section Économique et Commerciale, aurait pour objet de les perfectionner dans l'examen des problèmes concrets qui se posent, soit aux directeurs d'entreprises, soit aux responsables de l'Économie nationale. La seconde viserait les animateurs affectés par l'Administration au développement régional et local et leur inculquerait les connaissances fondamentales sur lesquelles doit s'appuyer leur action (ceci à la demande même du Ministère du Développement du Sénégal). Il entre aussi dans 